\title{
Super-strong and Intrinsically Fluorescent Silkworm Silk from Carbon Nanodots Feeding
}

Cite as

Nano-Micro Lett.

(2019) 11:75

Received: 25 June 2019

Accepted: 16 August 2019

Published online: 11 September 2019

(C) The Author(s) 2019

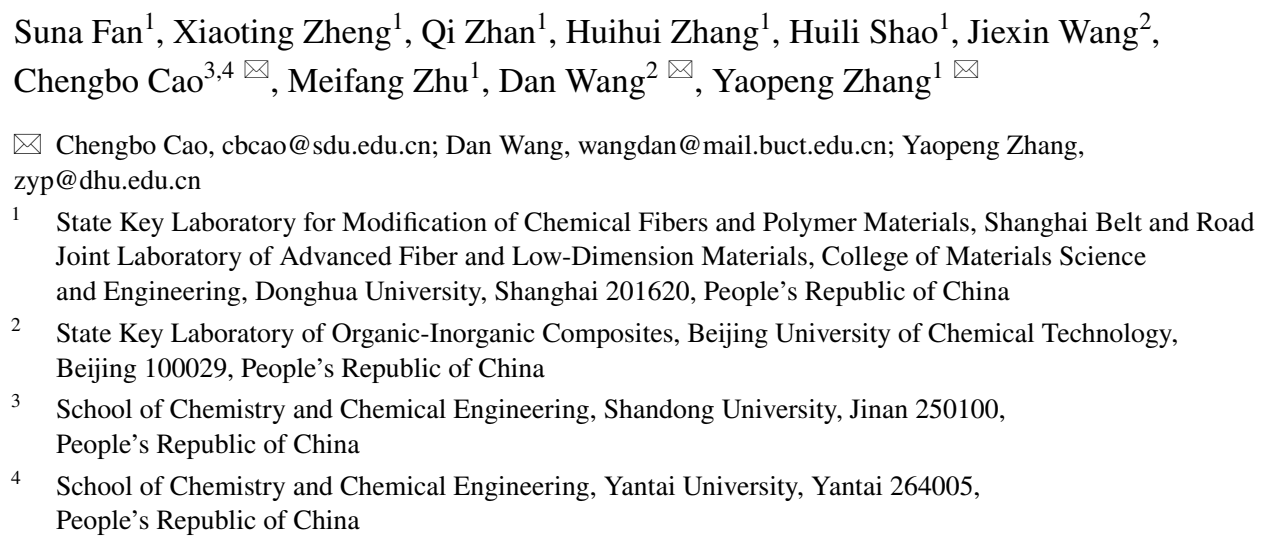

\section{HIGHLIGHTS}

- Intrinsically, super-strong fluorescent silk was fabricated via feeding Bombyx mori silkworms with carbon nanodots.

- The multi-functional silks showed no cytotoxicity to Schwann cells and exhibited great potential in bioimaging.

- The reinforcing mechanism of multi-functional silks was proposed.

\begin{abstract}
Fluorescent silk is fundamentally important for the development of future tissue engineering scaffolds. Despite great progress in the preparation of a variety of colored silks, fluorescent silk with enhanced mechanical properties has yet to be explored. In this study, we report on the fabrication of intrinsically super-strong fluorescent silk by feeding Bombyx mori silkworm carbon nanodots (CNDs). The CNDs were incorporated into silk fibroin, hindering the conformation transformation, confining crystallization, and inducing orientation of mesophase. The resultant silk exhibited super-strong mechanical properties with breaking strength of $521.9 \pm 82.7 \mathrm{MPa}$ and breaking elongation of $19.2 \pm 4.3 \%$, improvements of $55.1 \%$ and $53.6 \%$, respectively, in comparison with regular silk. The CNDs-reinforced silk displayed intrinsic blue fluorescence when exposed to $405 \mathrm{~nm}$

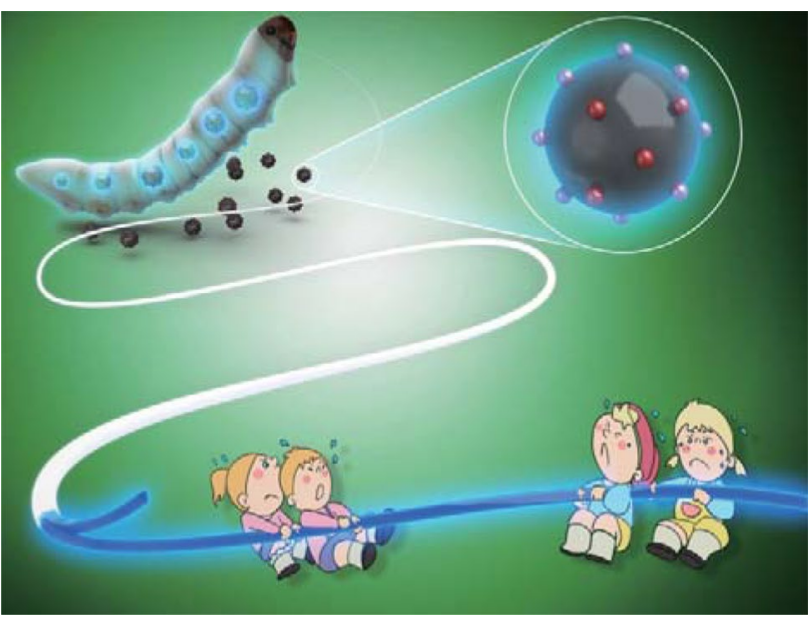
laser and exhibited no cytotoxic effect on cells, suggesting that multi-functional silks would be potentially useful in bioimaging and other applications.
\end{abstract}

KEYWORDS Silkworm silk; Carbon nanodot; Mechanical property; Fluorescent silk; Feeding method 


\section{Introduction}

Fluorescent silk, one of the novel natural functional biomaterials, is gaining enormous attention due to its great potential for biomedical and intelligent textile-related applications [1-5]. It has been reported that fluorescence can be imparted through the incorporation of various organic dyes and inorganic nanoparticles into silk through post-dyeing of naturally produced silk [6, 7]. This method inevitably requires harsh post-processing conditions and complex procedures, which may devastate the original properties of the silk. Alternatively, modification of silkworm genes to obtain intrinsically fluorescent silk has been reported [8-11]. This technique has great potential for the production of bio-functional silk in the future, especially as the mass production of spider silk can be achieved through targeted gene replacement in Bombyx mori [12]. Furthermore, a recent study has reported the direct production of intrinsically fluorescent silk from silkworms fed on a diet containing dye [13]. Compared with other methods, feeding method is considerably more convenient and environmentally friendly since it eliminates all external dyeing processes and reduces the use of the resources associated with it.

Besides its fluorescent capability, the properties required for fluorescent silk for use in biomedical scaffolds and diagnostic and therapeutic devices are excellent mechanical properties and lack of cytotoxicity. To date, to the best of our knowledge, the majority of fluorescent silk only restores the original mechanical properties of silk, which is still much weaker than that of spider dragline silk or other reinforced silk [14, 15]. Super-strong silk with intrinsically fluorescence has not yet been reported.

In the present study, we have reported the fabrication of reinforced and fluorescent multi-functional silk produced directly by silkworms, that is, reinforced intrinsically fluorescent silk, and studied its physical properties. Based on the reinforcing mechanism, we hypothesize that the fluorescent material added to the silkworms' diet should present a number of functional groups that would be able to interact with silk fibroin, but otherwise have little effect on the original structure of silk fibroin. As potential candidates, it was found that carbon nanodots (CNDs) have the desired structure [16, 17]. Furthermore, as a nanodye, CNDs disperse homogeneously in the diet, favoring in vivo uptake to form high-quality silk [18]. In addition, CNDs are a new class of fluorescent materials that has attracted much attention for applications in biomedical field owing to their adjustable parameters, good fluorescence stability, and excellent biocompatibility [19-21].

\section{Experimental}

\subsection{Carbon Nanodots}

Carbon nanodots (CNDs) were provided by Professor D. Wang, Beijing University of Chemical Technology, and prepared using a modified hydrothermal method with citric acid and ethylenediamine as precursors [22]. The CNDs exhibited excellent dispersibility in aqueous solution and had diameters ranging from 1 to $5 \mathrm{~nm}$ (Fig. S2).

\subsection{Preparation of CNDs-Modified Artificial Diet}

The Bombyx mori eggs were provided by the Sericultural Research Institute of Guangxi, China. The artificial silkworm diet was purchased from Shandong Sericultural Research Institute, China. The preparation of artificial diet is the same as our previous work [23]. Dry diet power was mixed uniformly with CNDs in aqueous suspension, followed by microwaving for $5 \mathrm{~min}$, and then pressed into wafer. The mass fraction ratio of CNDs/dry diet powder was $0.00,0.75,1.00$, and $1.25 \%$.

\subsection{Raising of Silkworm}

The Bombyx mori silkworms were raised in a climatic chamber. The humidity and temperature in different growth periods were controlled accurately as previously reported by Cai et al. [23]. All silkworm larvae were fed with normal artificial diet prior to the second day of the fifth instar. A total of 100 silkworms were then allocated equally into four groups. Three groups were fed a CNDs-modified diet from the second day of the fifth instar to the start of spinning since the growth of silk gland and biosynthesis and secretion of silk fibroin occurred mostly during this period [24]. The other group was fed a normal artificial diet over the whole study. Finally, the survival rate of each group was up to $95 \%$. The silkworms and resultant silks obtained were 
termed CNDs- $0.75 \%$, CNDs- $1 \%$, CNDs- $1.25 \%$, and control, respectively.

The normal artificial diet consisted of mulberry leaf powder $(38.4 \%)$, defatted soybean powder $(36.9 \%)$, corn powder $(9.0 \%)$, agar powder $(5.0 \%)$, green branches and petioles powder (5.0\%), other trace substances, including vitamin $\mathrm{C}$, vitamin B complex, choline chloride, and citric acid.

\subsection{Cocoon Degumming}

Prior to degumming, the obtained cocoons were dried at $100{ }^{\circ} \mathrm{C}$ for $60 \mathrm{~min}$ and at $80^{\circ} \mathrm{C}$ for $180 \mathrm{~min}$ in a vacuum drying oven. The cocoons were then degummed three times in boiling $0.5 \mathrm{wt} \% \mathrm{Na}_{2} \mathrm{CO}_{3}$ aqueous solution for $30 \mathrm{~min}$ and rinsed with distilled water. Finally, the degummed silks were dried at room temperature.

\subsection{Cell Culture and Characterization}

Silk scaffolds were constructed by winding silk fibers around a hollow plastic frame (Fig. 4a), followed by degumming. Silk scaffolds were placed into 24-well plate and sterilized using $75 \%$ (v/v) ethanol aqueous solution and UV light, followed by washing with phosphate-buffered saline (PBS) three times. Schwann cells (SCs) were obtained from the Institute of Biochemistry and Cell Biology of the Chinese Academy of Sciences (Shanghai, China). They were seeded on scaffolds at a density of $1 \times 10^{4}$ cells per well and cultured in Dulbecco's modified Eagle medium (DMEM) supplemented with $10 \%$ (v/v) fetal calf serum and $10 \%$ (v/v) double antibody in a cell incubator at $37{ }^{\circ} \mathrm{C}$ in an atmosphere containing 5\% $\mathrm{CO}_{2}$. Culture medium was exchanged every 2 days. Cell viability was evaluated using 3-[4,5-dimethyl2-thiazolyl]-2,5-diphenyl-2H-tetrazolium bromide (MTT) method after seeding for 2, 4, and 6 days. After 4 days, scanning electron microscopy (SEM, S-4800, Japan) and laser scanning confocal microscopy (LSCM, TCS SP5, Germany) were used to study the morphology of SCs. For observation by SEM, SCs seeded on scaffolds (SCs/scaffolds) were washed with PBS three times and then fixed in $2.5 \%$ paraformaldehyde at $4{ }^{\circ} \mathrm{C}$ for $2 \mathrm{~h}$. After removing paraformaldehyde, SCs/scaffolds were washed in PBS three times again, followed by dehydration through a gradient of ethanol aqueous solutions $(30,50,70,75,80,90$, and 100 vol\%). Finally, the resulting SCs/scaffolds were freeze-dried in tert-butanol solution. SEM images were obtained at $10 \mathrm{kV}$. For observation by LSCM, SCs/scaffolds were fixed in $4.0 \%$ paraformaldehyde for $10 \mathrm{~min}$, followed by washing in PBS three times and permeabilizing using $0.1 \%$ Triton $\mathrm{X}-100$ solution for $5 \mathrm{~min}$. The samples were blocked in $1.0 \% \mathrm{BSA}$ solution for $20 \mathrm{~min}$. Finally, SCs/scaffolds were incubated with phalloidin solution for $30 \mathrm{~min}$. The laser wavelength was $405 \mathrm{~nm}$.

\subsection{Structural Characterization}

Silk surface morphology was imaged using a Hitachi S-3000 N scanning electron microscope (SEM). The CNDs were observed using a JEM-2100 transmission electron microscope (TEM) operated at $200 \mathrm{kV}$. Photoluminescence (PL) spectra following excitation using a $370 \mathrm{~nm}$ laser were collected on JASCO FP-6600 PL instrument. The CLSM (confocal laser scanning microscopy) images of the degummed silk were obtained after excitation with a $405 \mathrm{~nm}$ laser and acquired on TCS SP5 LSCM. 3D CLSM images were reconstructed using Imaris software.

Fourier transform infrared (FTIR) spectra were recorded on a Nicolet 6700 Fourier transform spectrometer attenuated total reflectance (ATR) accessory. Quantitative analysis of secondary structure was conducted by spectra deconvolution of amide I band [25, 26].

Synchrotron radiation wide-angle X-ray diffraction (SRWAXD) was performed on BL15U1 beamline at Shanghai Synchrotron Radiation Facility. The wavelength $(\lambda)$ and the spot size of the X-ray were $0.07746 \mathrm{~nm}$ and $3 \times 2 \mu \mathrm{m}^{2}$, respectively. FIT2D (V12.077) software and Peakfit (V4.12) software were utilized to process data obtained from these analyses. The process method was described in detail in our previous work [27].

The diameter of degummed silk was measured using an Olympus BX-51 optical microscope. However, the cross section of silk was irregular, and a more accurate diameter was confirmed through a comparison of the method described above and typical weight/length method, as detailed in Ref. [23]. For each sample, more than 15 single silk fibers were measured. Subsequently, the mechanical properties of silks were measured on Instron 5565 at $25^{\circ} \mathrm{C}$ and $(45 \pm 5) \%$ relative humidity. The extension rate and the gauge length were $2 \mathrm{~mm} \mathrm{~min}^{-1}$ and $10 \mathrm{~mm}$, respectively. 


\section{Results and Discussion}

\subsection{Growth of Silkworms and Physical Properties of Cocoons}

The Bombyx mori larval silkworms were raised in the climatic chamber by feeding with CNDs-modified diet from the second day of the fifth instar to the start of spinning, in order to produce multi-functional silks. The diets containing different content CNDs were prepared as previously described [23]. The cocoons and silk fibers obtained by feeding diets with CNDs concentration of $0,0.75,1.00$, and $1.25 \mathrm{wt} \%$ were named by control, CNDs-0.75, CNDs-1.00, and CNDs-1.25, respectively. All silk larvae had a similar weight over the duration of fifth instar (Fig. 1e) with no differences between the control mature larva and those fed with CNDs-modified diet. This indicated that modified diets prepared in this study were safe for silkworm. In addition, the cocoons obtained in this way exhibited similar colors and sizes (Fig. 1a-d). After drying, the cocoons were boiled in $0.5 \mathrm{wt} \% \mathrm{Na}_{2} \mathrm{CO}_{3}$ aqueous solution to remove sericin so as to obtain degummed silks, used for subsequent characterizations. Figure 1a-d demonstrates that degummed silk exhibited similar smooth morphology with a diameter of $7 \mu \mathrm{m}$. No any CNDs were observed, illustrating that direct feeding with modified diet had no apparent effects on silk morphology. This may be attributed to the small number and excellent water dispersibility of CNDs (Fig. S2), a
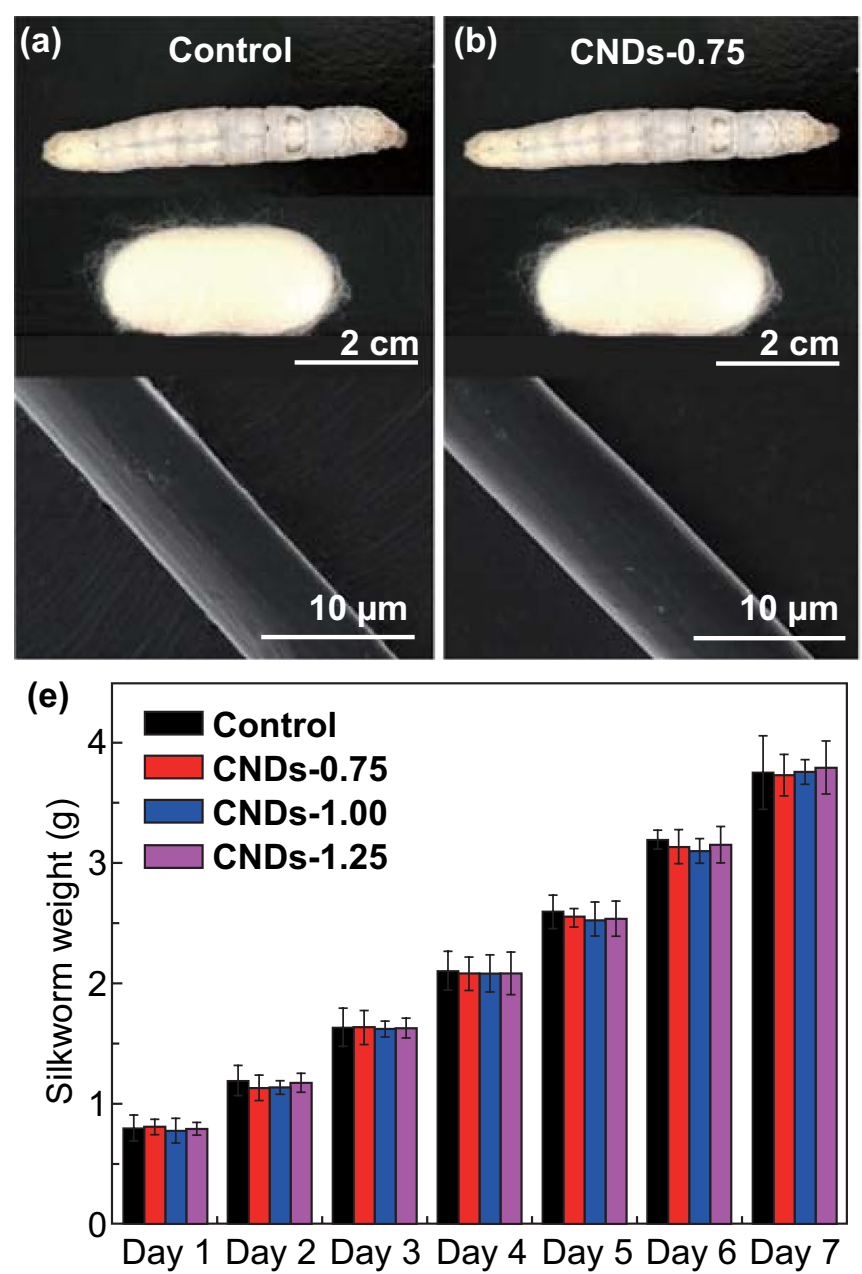
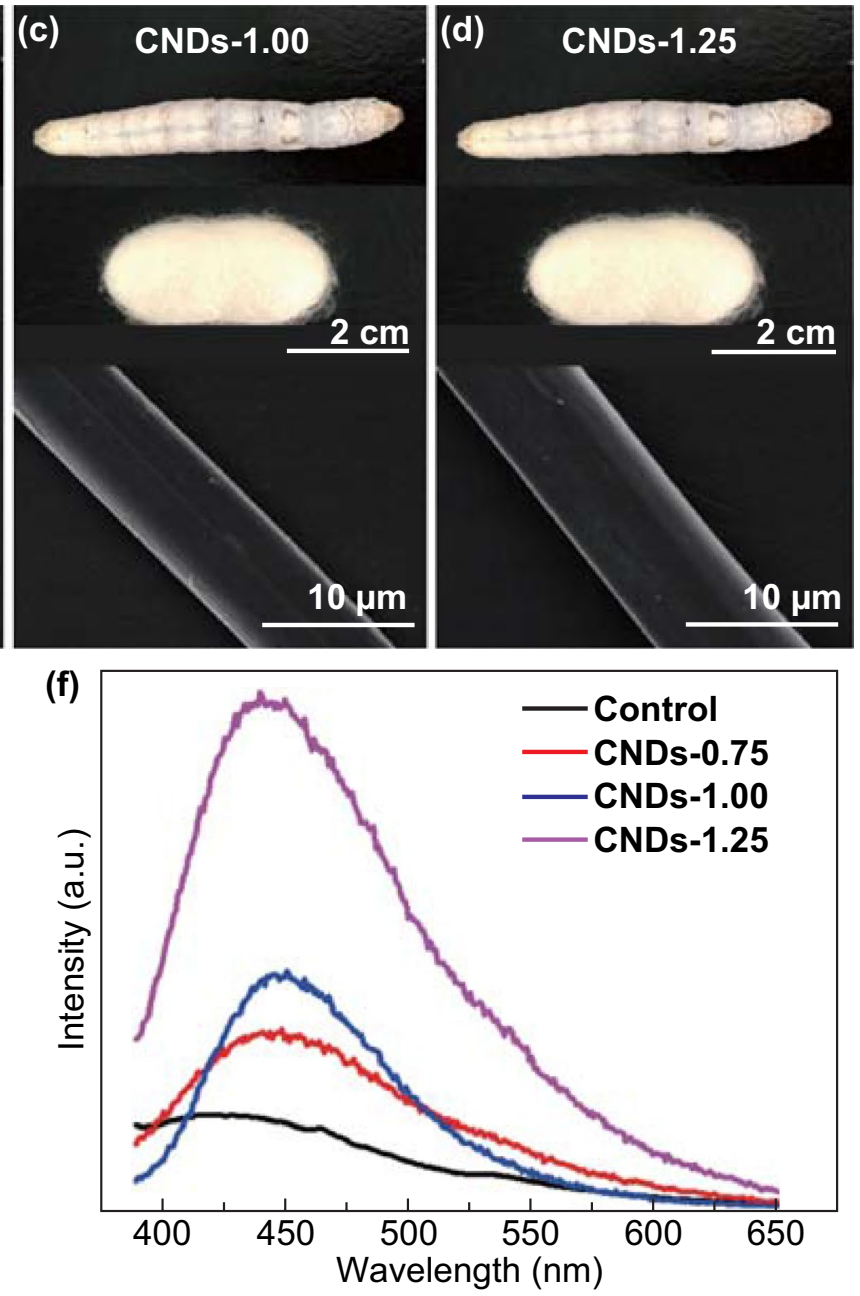

Fig. 1 Preparation of multi-functional silk from silkworms fed CNDs. a-d Photographs of mature larvae fed different diets, corresponding cocoons and degummed silks. e Weight of silkworm larvae of the fifth instar from first to seventh day. f PL spectra of degummed silks measured using an excitation wavelength of $370 \mathrm{~nm}$ 
characteristic difference from graphene, $\mathrm{TiO}_{2}$, and other inorganic nanoparticles.

\subsection{Fluorescent Properties of CNDs-Modified Silks}

An important observation was that just a small quantity of CNDs could endow the silk with unique properties. As shown in the PL spectra (Fig. 1f), the modified silks exhibited a strong emission peak at $450 \mathrm{~nm}$, the intensity of which increased as the concentration of CNDs increased. The control silks demonstrated only a weak but broad emission peak centered at $420 \mathrm{~nm}$. The CNDs in the present study exhibited a strong emission peak centered at $450 \mathrm{~nm}$ (Fig. 2f); hence, we concluded that the fluorescent property originated from the CNDs, and silkworms could take in a certain quantity of CNDs.

As a result, all degummed silks, except the controls, exhibited an intrinsic homogeneous blue fluorescence at an excitation wavelength of $405 \mathrm{~nm}$ (Figs. 2b-e and S3). The CNDs-1.25 silk exhibited the brightest blue fluorescence, consistent with the PL spectra (Fig. 1f). It is worth noting that samples with blue fluorescence were degummed silk rather than cocoon, indicating that the fluorescence originated from silk fibroin brin. This was different from the naturally colored silk produced by wild silkworms, the color of which emanated from sericin rather than fibroin, and was lost after degumming [13].

In order to further ascertain the origin of blue fluorescence of the silk, the silk glands of mature larvae fed with different diets were compared under UV lamp. The whole silk glands of control silkworm exhibited a light yellowish green (Fig. 2b') fluorescence, while the middle and anterior silk gland of CNDs-0.75 silkworm fluoresced light blue although the color of the fluorescence from the posterior silk gland (Fig. 2c') was similar to that of control silkworm. As the content of CNDs reached up to 1 and $1.25 \mathrm{wt} \%$, the whole silk glands fluoresced deep blue (Fig. 2d'-e') and
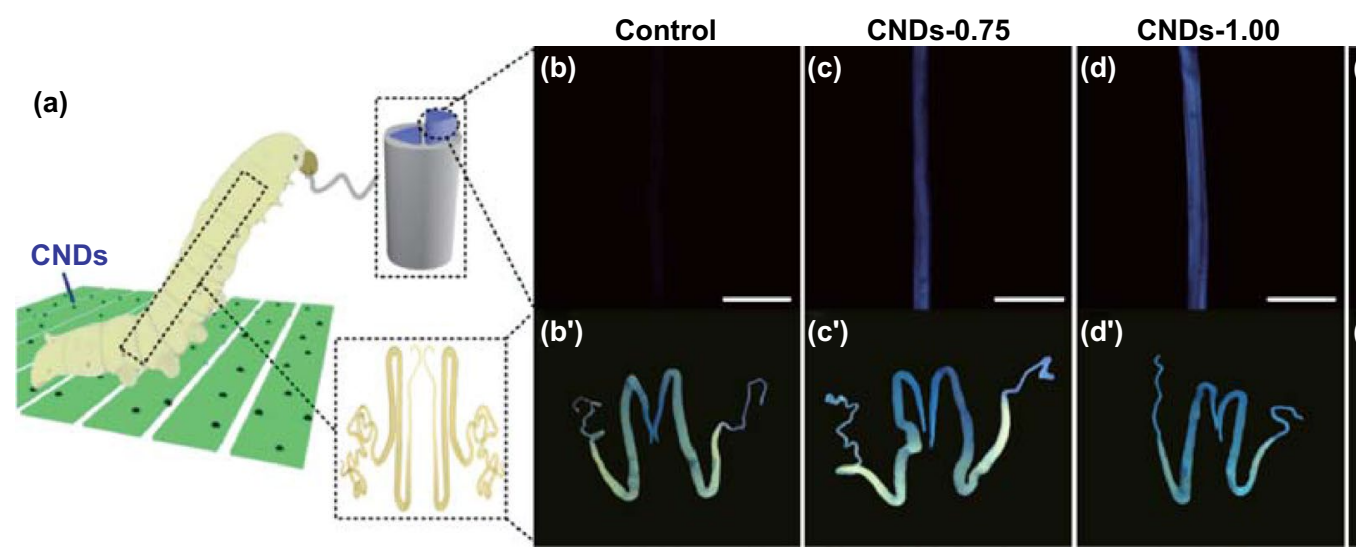

CNDs-1.25
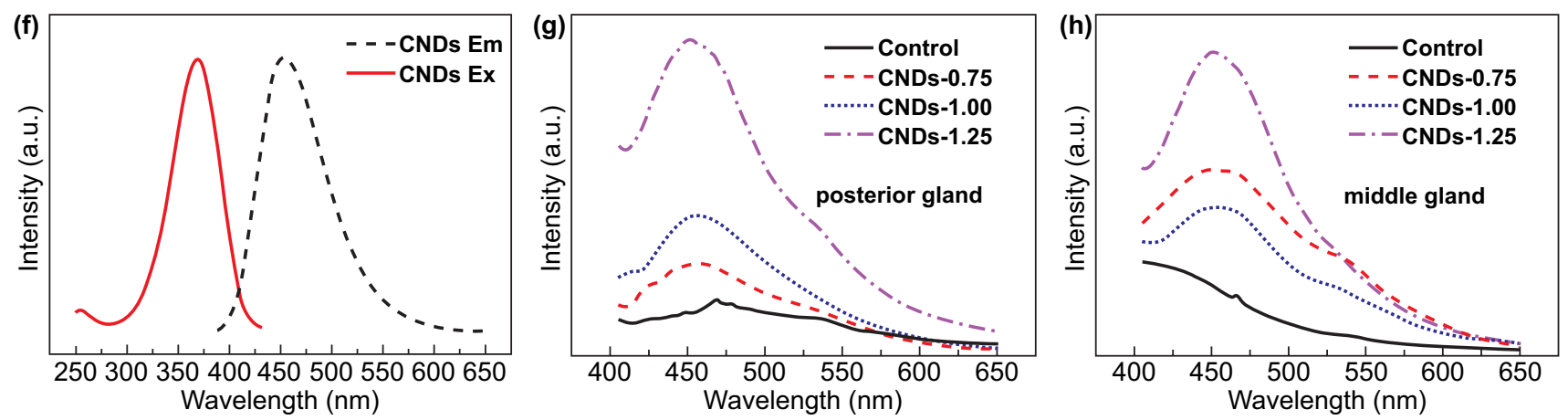

Fig. 2 Influence of CNDs additives on the intrinsic fluorescent intensity of silk. a Schematic of silkworm fed a CNDs-modified diet and the corresponding silk gland and silk fiber. b-e Confocal laser scanning microscopy images of degummed silks (measured with an excitation wavelength of $405 \mathrm{~nm}$ ). Scale bar represents $50 \mu \mathrm{m}$. $\mathbf{b}^{\prime}-\mathbf{e}^{\prime}$ Ultraviolet (UV) pictures of silk glands of mature larvae measured under a UV lamp. b, $\mathbf{b}^{\prime}$ Control, $\mathbf{c}, \mathbf{c}^{\prime}$ CNDs- $0.75 \%, \mathbf{d}, \mathbf{d}^{\prime}$ CNDs-1\%, e, $\mathbf{e}^{\prime}$ CNDs-1.25\%. f-h PL spectra of (f) CNDs, (g) posterior and (h) middle silk glands with an excitation wavelength of $370 \mathrm{~nm}$ 
further confirmed by the PL spectra of middle and posterior silk glands (Fig. 2g-h). In addition, the color of the fluorescence of glands became bluer with the concentration of silk fibroin from posterior silk gland to anterior division of middle silk gland. Asukura [28] pointed out that silk fibroin was polymerized in posterior silk gland. These observations illustrated again that CNDs were most likely within the silk fibroin and were maintained a certain content during the process of concentration and spinning, causing the modified silk to become intrinsically blue fluorescent.

\subsection{Mechanical Properties of CNDs-Modified Silks}

An important fact is that the CNDs-modified silks exhibited dramatically improved mechanical properties compared with control silk (Table 1 and Fig. S4). The breaking strength and elongation of $521.9 \mathrm{MPa}$ and $19.2 \%$ for CNDs- 1.25 silk significantly exceed those of control silk with $336.5 \mathrm{MPa}$ and $12.5 \%$, indicating that super-strong fluorescent silks could be fabricated using even small quantities of CNDs. Hence, $1.25 \mathrm{wt} \%$ was chosen as the maximum content of CNDs in this study to save the additional costs of further scale production and avoid any adverse effect of excessive CNDs on silkworms and silks [23]. Similar to other modified silks from silkworms fed silver nanoparticle [29], threonine [30], or nanohydroxyapatite powers [31], the mechanical properties of CNDs-modified silks were more variable than those of control silk. This may be attributed to the effect of exogenous addition on silkworm's spinning behavior including spinning speed, which significantly determines the performance of silk [32]. Detailed investigations are still required to answer this question further. Moreover, the multi-functional silk with intrinsic fluorescence and enhanced mechanical properties was reported here for the first time, differentiating them from other fluorescent silk [9, 13]. Note that the mechanical properties of silks increased as CNDs content increased over the range $0.75-1.25 \mathrm{wt} \%$, different from the results of silk modified by graphene [33], and provided the potential to modulate the properties for different applications. In addition, the mechanical properties of the silks demonstrated that they were stronger than other reported fluorescent silk [9, 13], although it was still lower than some reported silks $[34,35]$. It should be noted that the environment in which the silkworms are raised, the process for degumming and approaches for testing all add to the variability of properties measured. Hence, in this study we only compared the properties and discussed the structures of silks fabricated within the same conditions.

\subsection{Reinforcing Mechanism of CNDs-Modified Silks}

We utilized FTIR to study the secondary structures of silk, strongly related to its mechanical properties. A peak at $1695 \mathrm{~cm}^{-1}$ was considered attributable to $\beta$-turn conformation [36], and the peaks at 1623 and $1230 \mathrm{~cm}^{-1}$ were related to $\beta$-sheet conformation [37]. The peak centered at $1265 \mathrm{~cm}^{-1}$ was assigned to a random coil/ $\alpha$-helix conformation [25]. The above characteristic peaks of silk fibroin were observed in all degummed silks, with no significant differences observed, suggesting that there were no strong covalent interactions between silk fibroin and CNDs. However, compared with control silk, the CNDs-modified silks contained a greater number of chains in random coil/ $\alpha$-helix conformation and fewer with $\beta$-sheet conformation (Figs. $3 b$ and S5). This may be attributed to the abundant carboxyl and hydroxyl on the surface of CNDs (Fig. S6), favoring

Table 1 Mechanical properties of intrinsically fluorescent degummed silk fibers

\begin{tabular}{lllll}
\hline Sample & Breaking strength (MPa) & Breaking elongation (\%) & $\begin{array}{c}\text { Modulus (GPa) } \\
\text { Breaking } \\
\text { energy } \\
\left.(\mathrm{kJ} \mathrm{kg})^{-1}\right)\end{array}$ \\
\hline Control & $336.5 \pm 27.0$ & $12.5 \pm 2.2$ & $7.3 \pm 1.7$ & $21.6 \pm 5.0$ \\
CNDs-0.75 & $390.4 \pm 91.5$ & $15.3 \pm 3.2$ & $8.3 \pm 2.7$ & $30.4 \pm 7.5$ \\
CNDs-1.00 & $479.4 \pm 86.7$ & $15.8 \pm 3.1$ & $8.4 \pm 2.5$ & $36.8 \pm 7.3$ \\
CNDs-1.25 & $\mathbf{5 2 1 . 9} \pm \mathbf{8 2 . 7}$ & $\mathbf{1 9 . 2} \pm \mathbf{4 . 3}$ & $\mathbf{8 . 9} \pm \mathbf{2 . 2}$ & $\mathbf{5 1 . 4} \pm \mathbf{1 4 . 3}$ \\
Transgenic silk [9] & $419.97 \pm 20.04$ & $21.02 \pm 1.49$ & - & - \\
Silk from silkworm fed on & $406-454$ & $23.7-26.5$ & & - \\
rhodamine [13] & & & & \\
\hline
\end{tabular}



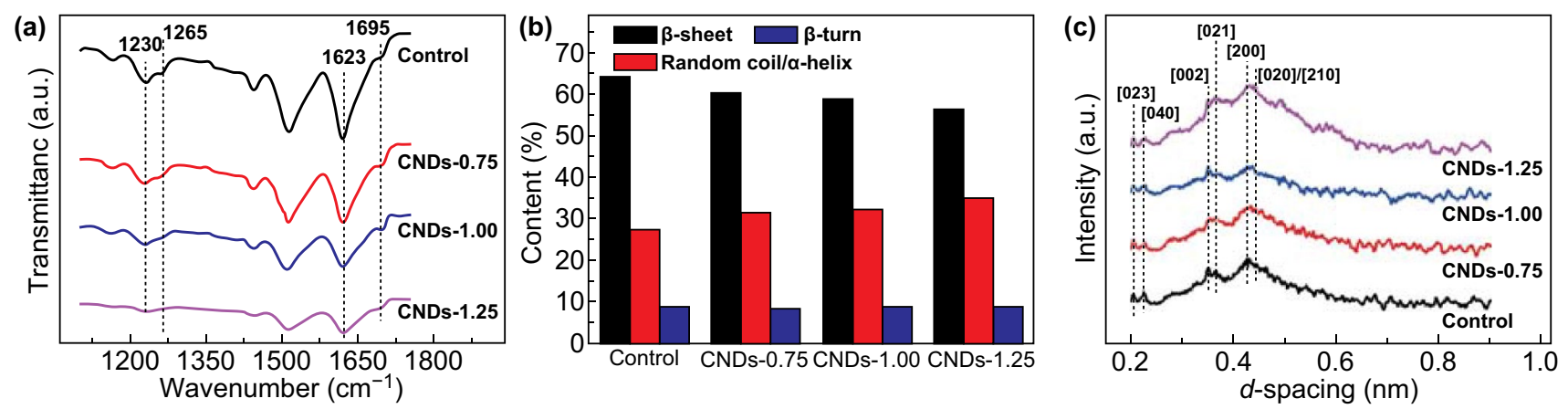

(d)

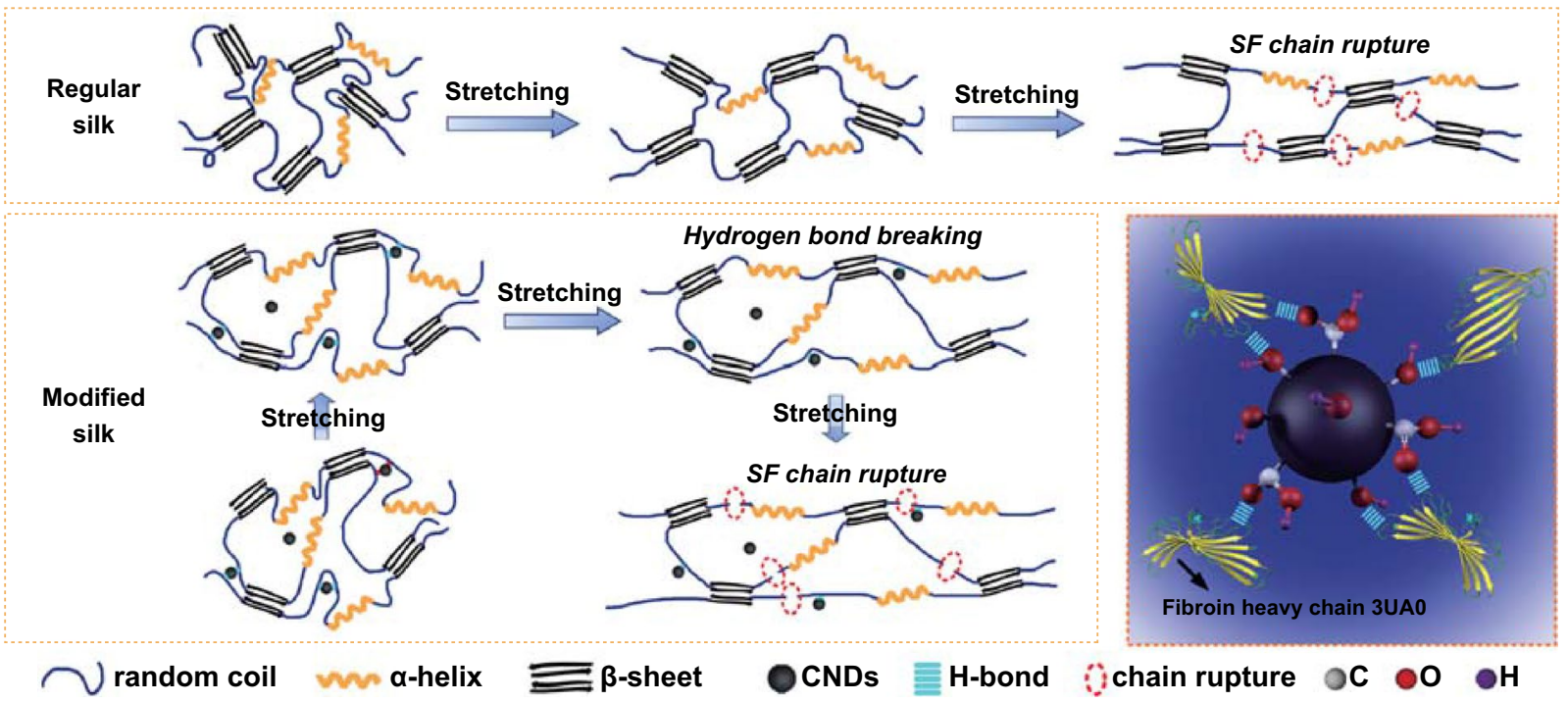

Fig. 3 Crystalline structure and proposed reinforcing mechanism of multi-functional silk from silkworms fed CNDs. a-c FTIR spectra (a), content of secondary structures (b) obtained based on the amide I band in FTIR spectra, and 1D-WAXD patterns (c) of degummed silks. d Schematic diagram illustrating reinforcing mechanism of silks by incorporation of CNDs

the formation of hydrogen bonds with amino groups of silk fibroin, hindering the transformation from random coil/ $\alpha$ helix to $\beta$-sheets.

The crystalline structure and orientation of silk fibers can be evaluated using synchrotron radiation wide-angle X-ray diffraction (SR-WAXD) (Figs. 3c and S7). All the silks exhibited the principal crystalline peaks of [002], [021],
[200], and [020]/[210] lattice planes at d-spacing of 0.35 , $0.37,0.43$, and $0.45 \mathrm{~nm}$, with no remarkable differences, as shown in 1D WAXD. However, quantitative analyses of 1D WAXD revealed that the crystallinity of the modified silks was lower than control silks and decreased with increasing content of CNDs (Table 2), consistent with the FTIR result and previous reports [23, 38]. Based on "hydrogen

Table 2 Herman's orientation function and crystallinity parameters of degummed silks

\begin{tabular}{|c|c|c|c|c|c|c|c|}
\hline \multirow[t]{2}{*}{ Sample } & \multirow[t]{2}{*}{$f_{\text {crystal }}$} & \multirow[t]{2}{*}{$f_{\text {mesophase }}$} & \multirow[t]{2}{*}{ Crystallinity (\%) } & \multirow{2}{*}{$\begin{array}{l}\text { Mesophasecontent } \\
(\%)\end{array}$} & \multicolumn{3}{|c|}{ Crystallite size (nm) } \\
\hline & & & & & $(200) /(210)$ & $(020)$ & (002) \\
\hline Control & 0.9699 & 0.8579 & 49.3 & 13.1 & 5.0 & 3.1 & 10.4 \\
\hline CNDs- 0.75 & 0.9680 & 0.8587 & 45.8 & 14.5 & 4.4 & 3.8 & 9.3 \\
\hline CNDs- 1.00 & 0.9684 & 0.8613 & 44.5 & 14.9 & 4.1 & 3.9 & 9.7 \\
\hline CNDs- 1.25 & 0.9677 & 0.8709 & 43.1 & 15.7 & 4.2 & 3.3 & 9.2 \\
\hline
\end{tabular}


bond barrier" effect [39] and nanoconfined crystallization as proposed by Pan et al. [27], the hydrogen bonds between CNDs and silk fibroin would have been expected to hinder the motion of silk fibroin, resulting in more amorphous. The $\beta$-sheet crystalline structure in silk belongs to the orthorhombic system [40], and the crystallite size along $a$, $b$, and $c$ directions is determined from [200]/[210], [020], and [002] planes. Here, the lattice axes $a$ and $c$ are perpendicular, and along to silk fibroin chains, the lattice axis $b$ is perpendicular to the $\beta$-sheet. The crystallite sizes of CNDsmodified silks in the $a$ and $c$ directions were slightly smaller than those of control silk. However, this was contrary to the result measured in the $b$ direction. In addition, the crystalline volume evaluated by $L_{a} \times L_{b} \times L_{c}$ [41] decreased with increasing the content of CNDs, significantly different from the modified silks from silkworms fed $\mathrm{TiO}_{2}$ [23]. This might be explained by differences in the size and dispersibility of $\mathrm{CNDs}_{\text {and }} \mathrm{TiO}_{2}$. At the same addition, compared with $\mathrm{TiO}_{2}$ with poor dispersibility and a larger size $(20-50 \mathrm{~nm})$, the smaller-sized CNDs (1-5 nm, Fig. S2) could homogeneously disperse over every segment of silk gland (Fig. $2 c^{\prime}-e^{\prime}$ ) and move along the protein molecules during the process of spinning. Hence, hydrogen bond interactions between silk fibroin and CNDs acted as a "cross-linked knot" and hindered the movement of molecular chains, confining crystallization of the silk fibroin. This tendency had already been observed in other polymers due to the efficient suppression of the crystal extension at higher concentration of additives $[42,43]$. Furthermore, the smaller size and better mobility of CNDs favored the arrangement of molecular chains and phases that resulted in a comparable crystal orientation and higher mesophase orientation (Table 2), which included the oriented amorphous and interface zones between silk fibroin and CNDs [38, 44].

Based on the observation above, we hypothesize that the addition of CNDs would hinder the transformation of conformation, confine the crystallization, and induce orientation of mesophase. Those factors have played an important role in reinforcing the mechanical properties. Moreover, we should note that the addition of CNDs implied providing strong and stiff nanomaterial for the silk [45], further enhancing the modified silk. The proposed reinforcing mechanism is shown in Fig. 3d. Upon stretching, easily movable chains in random coil/ $\alpha$-helix conformation in the amorphous phase were first to deform. Meanwhile, the spherical morphology, nanometer size scale, and intensive hydrogen bond interactions caused the CNDs to move with protein chains [46], in turn providing more space for chains to move. This collaborative mobility endowed larger elongation to the modified silk fibers. As deformation increased, the relatively weak hydrogen bonds between CNDs and silk fibroin were broken first and dissipated energy [47]. In addition, the CNDs themselves provided stiffness and induced a transfer of stress from the silk to CNDs due to the nanofiller effect $[45,48]$. Furthermore, the more content and higher orientation of the mesophase further reinforced the mechanical properties of CNDs-modified silk fibers.

\subsection{Non-cytotoxicity and Application of CNDs-Modified Silks}

As a natural biomaterial, silk from normal silkworm has been widely used as tissue engineering scaffold. In this study, we constructed silk scaffolds by wrapping the fibers around a hollow plastic frame in order to culture SCs (Fig. 4a). After seeding for 2 days, the optical density (OD) value of SCs on different substrates was similar (Fig. 4b). After 4 or 6 days, SCs displayed higher proliferation on the silk scaffolds than coverslips, but no significant difference was observed between the control and modified scaffolds (Fig. 4c, d). This indicated that the non-cytotoxicity of natural silk was preserved in multi-functional silk scaffolds modified with CNDs and further confirmed by LSCM images of SCs stained with phalloidin (Fig. 4c' $\mathrm{c}^{\prime}, \mathrm{d}^{\prime}$ ). Furthermore, an important feature of fluorescent silk in tissue scaffolds is the improved visualization of cells. SCs which were in directly contact with fluorescent silks appeared bright pink, while other SCs appeared red. The blue fluorescence of silks also allowed more convenient monitoring of scaffold degradation. Taken together, CNDs-modified silks with excellent mechanical property, intrinsically fluorescent property, and non-cytotoxicity might motivate to open up new applications in tissue engineering field.

\section{Conclusions}

In summary, multi-functional silks with reinforced mechanical properties, intrinsically fluorescence, and non-cytotoxicity can be produced simply using a simple in vivo modification method. The breaking strength and elongation of CNDs-1.25 fluorescent silk reached up to 
(a)

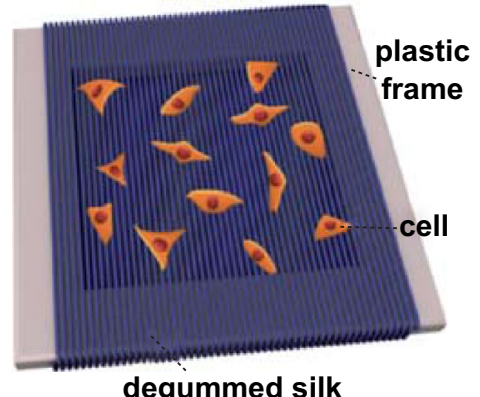

degummed silk

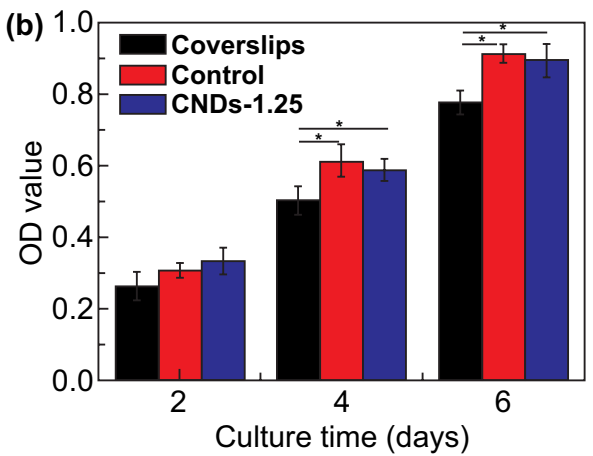

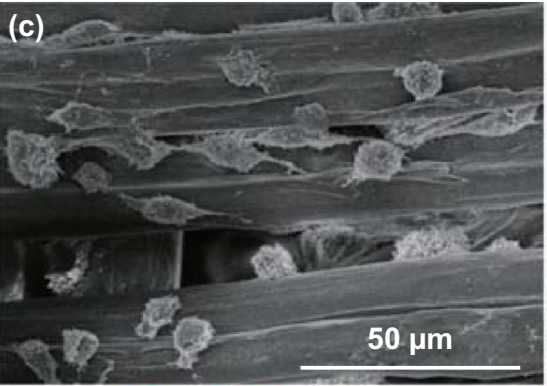
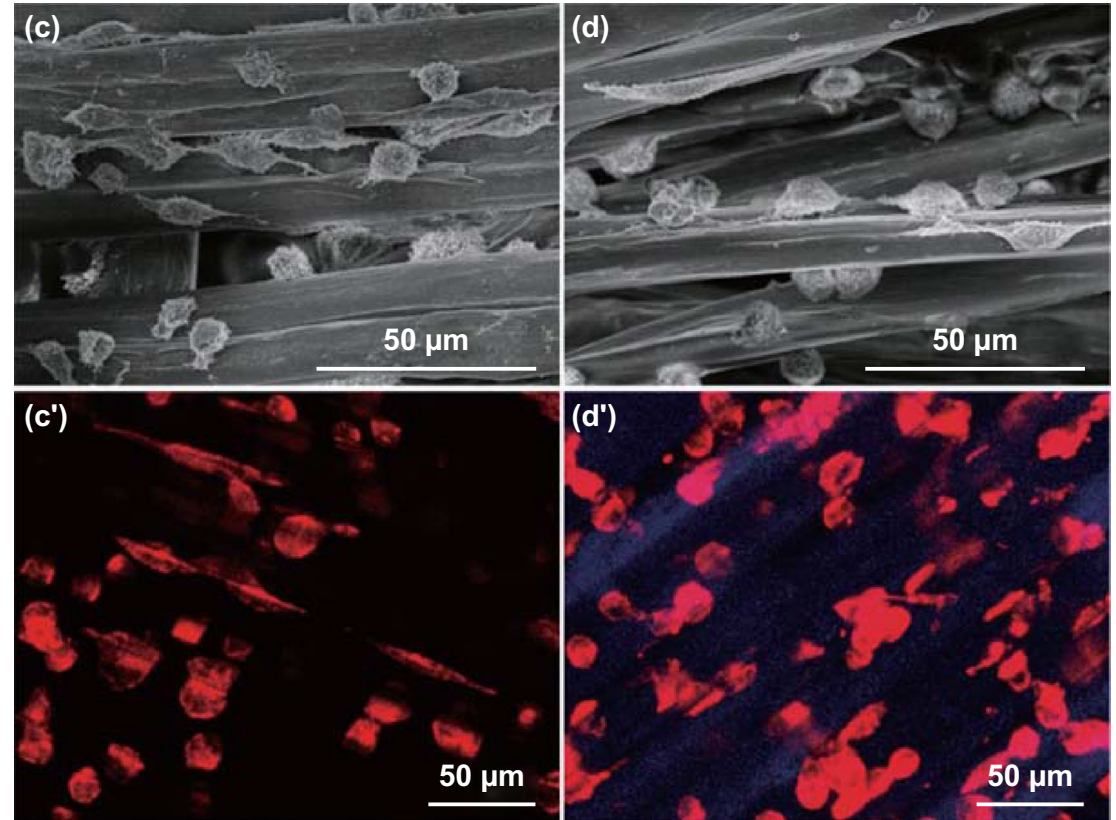

Fig. 4 Growth and proliferation of Schwann cells (SCs) on multi-functional silks. a SCs cultured on silk scaffolds, constructed by wrapping silk around a hollow plastic frame. b OD value of SCs cultured on different substrates (coverslips, control, and CNDs-1.25 silk scaffolds) after 2, 4, 6 days. $\mathbf{c}-\mathbf{d}^{\prime}$ SEM images $(\mathbf{c}, \mathbf{d})$ and LSCM images $\left(\mathbf{c}^{\prime}, \mathbf{d}^{\prime}\right)$ of SCs after culturing for 4 days on control $\left(\mathbf{c}, \mathbf{c}^{\prime}\right)$ and CNDs-1.25 silk scaffolds $\left(\mathbf{d}, \mathbf{d}^{\prime}\right)$

$521.9 \pm 82.7 \mathrm{MPa}$ and $19.2 \pm 4.3 \%$, respectively, considerably higher than that of regular silk and comparable to silks modified through post-treatment. This may be attributed to the hydrogen bonds between CNDs and silk fibroin, resulting in more random coil/ $\alpha$-helix structure, mesophase, and higher orientation. In addition, the nanosize and excellent dispersibility of CNDs were favored in the production of silks with a homogeneous blue fluorescence. It can be expected that large-scale production of multi-functional silks would be feasible and that the use of such silk as active scaffolds in tissue engineering would improve the functionalities of conventional biomaterials.

Acknowledgements This work was sponsored by the National Key Research and Development Program of China (2016YFA0201700, 2016YFA0201702), the Fundamental Research Funds for the Central Universities (2232019A3-06, 2232019D3-02), the National Key Research and Development Program of China (2018YFC1105800), the National Natural Science Foundation of China (21674018, 51903045), and the Shanghai Belt and Road
Joint Laboratory of Advanced Fiber and Low-Dimension Materials (18520750400).

Open Access This article is distributed under the terms of the Creative Commons Attribution 4.0 International License (http:// creativecommons.org/licenses/by/4.0/), which permits unrestricted use, distribution, and reproduction in any medium, provided you give appropriate credit to the original author(s) and the source, provide a link to the Creative Commons license, and indicate if changes were made.

Electronic supplementary material The online version of this article (https://doi.org/10.1007/s40820-019-0303-z) contains supplementary material, which is available to authorized users.

\section{References}

1. J.P. Mondia, J.J. Amsden, D. Lin, L.D. Negro, D.L. Kaplan, F.G. Omenetto, Rapid nanoimprinting of doped silk films for 
enhanced fluorescent emission. Adv. Mater. 22, 4596-4599 (2010). https://doi.org/10.1002/adma.201001238

2. N.C. Tansil, L.D. Koh, M. Han, Functional silk: colored and luminescent. Adv. Mater. 24, 1388-1397 (2012). https://doi. org/10.1002/adma.201104118

3. D.W. Kim, O.J. Lee, S.-W. Kim, C.S. Ki, J.R. Chao et al., Novel fabrication of fluorescent silk utilized in biotechnological and medical applications. Biomaterials 70, 48-56 (2015). https://doi.org/10.1016/j.biomaterials.2015.08.025

4. T.S. Kusurkar, I. Tandon, N.K. Sethy, K. Bhargava, S. Sarkar, S.K. Singh, M. Das, Fluorescent silk cocoon creating fluorescent diatom using a "water glass-fluorophore ferry". Sci. Rep. 3, 3290 (2013). https://doi.org/10.1038/srep03290

5. S.J. Ling, D.L. Kaplan, M.J. Buehler, Nanofibrils in nature and materials engineering. Nat. Rev. Mater. 3, 18016 (2018). https://doi.org/10.1038/natrevmats.2018.16

6. H. Somashekarappa, V. Annadurai, Sangappa, G. Subramanya, R. Somashekar, Structure-property relation in varieties of acid dye processed silk fibers. Mater. Lett. 53, 415420 (2002). https://doi.org/10.1016/S0167-577X(01)00517 $-1$

7. Y. Zhou, J. Zhang, R. Tang, J. Zhang, Simultaneous dyeing and functionalization of silk with three natural yellow dyes. Ind. Crops Prod. 64, 224-232 (2015). https://doi. org/10.1016/j.indcrop.2014.09.041

8. M. Tomita, H. Munetsuna, T. Sato, T. Adachi, R. Hino et al., Transgenic silkworms produce recombinant human type III procollagen in cocoons. Nat. Biotechnol. 21, 52-56 (2002). https://doi.org/10.1038/nbt771

9. T. Iizuka, H. Sezutsu, K.-I. Tatematsu, I. Kobayashi, N. Yonemura et al., Colored fluorescent silk made by transgenic silkworms. Adv. Funct. Mater. 23, 5232-5239 (2013). https ://doi.org/10.1002/adfm.201300365

10. J.W. Leem, S.H. Choi, S.R. Kim, S.W. Kim, K.H. Choi, Y.L. $\mathrm{Kim}$, Scalable and continuous nanomaterial integration with transgenic fibers for enhanced photoluminescence. Mater. Horiz. 4, 281-289 (2017). https://doi.org/10.1039/c6mh0 $0423 \mathrm{~g}$

11. S.W. Kim, E.Y. Yun, K.-H. Choi, S.R. Kim, S.W. Park, S.W. Kang, O.Y. Kwon, T.W. Goo, Construction of fluorescent red silk using fibroin H-chain expression system. J. Seric. Entomol. Sci. 50, 87-92 (2012). https://doi.org/10.7852/ jses.2012.50.2.87

12. J. Xu, Q. Dong, Y. Yu, B. Niu, D. Ji et al., Mass spider silk production through targeted gene replacement in Bombyx mori. PNAS USA 115, 8757-8762 (2018). https://doi. org/10.1073/pnas.1806805115

13. N.C. Tansil, Y. Li, C. Teng, S. Zhang, K. Win, X. Chen, X. Liu, M. Han, Intrinsically colored and luminescent silk. Adv. Mater. 23, 1463-1466 (2011). https://doi.org/10.1002/ adma. 201003860

14. S.W. Cranford, A. Tarakanova, N.M. Pugno, M.J. Buehler, Nonlinear material behaviour of spider silk yields robust webs. Nature 482, 72-76 (2012). https://doi.org/10.1038/ nature 10739
15. F.G. Omenetto, D.L. Kaplan, New opportunities for an ancient material. Science 329, 528-531 (2010). https://doi. org/10.1126/science. 1188936

16. S. Zhu, Q. Meng, L. Wang, J. Zhang, Y. Song et al., Highly photoluminescent carbon dots for multicolor patterning, sensors, and bioimaging. Angew. Chem. Int. Ed. 52, 3953-3957 (2013). https://doi.org/10.1002/anie.201300519

17. S.N. Baker, G.A. Baker, Luminescent carbon nanodots: emergent nanolights. Angew. Chem. Int. Ed. 49, 6726-6744 (2010). https://doi.org/10.1002/anie.200906623

18. H. Liu, T. Ye, C. Mao, Fluorescent carbon nanoparticles derived from candle soot. Angew. Chem. Int. Ed. 46, 64736475 (2007). https://doi.org/10.1002/anie.200701271

19. L. Wang, S. Zhu, H. Wang, S. Qu, Y. Zhang et al., Common origin of green luminescence in carbon nanodots and graphene quantum dots. ACS Nano 8, 2541-2547 (2014). https ://doi.org/10.1021/nn500368m

20. V. Strauss, K. Marsh, M.D. Kowal, M. El-Kady, R.B. Kaner, A simple route to porous graphene from carbon nanodots for supercapacitor applications. Adv. Mater. 30, 1704449 (2018). https://doi.org/10.1002/adma.201704449

21. A.M. Chizhik, S. Stein, M.O. Dekaliuk, C. Battle, W. Li et al., Super-resolution optical fluctuation bio-imaging with dual-color carbon nanodots. Nano Lett. 16, 237-242 (2015). https://doi.org/10.1021/acs.nanolett.5b03609

22. D. Wang, Z. Wang, Q. Zhan, Y. Pu, J. Wang, N.R. Foster, L. Dai, Facile and scalable preparation of fluorescent carbon dots for multifunctional applications. Engineering 3, 402-408 (2017). https://doi.org/10.1016/j.eng.2017.03.014

23. L. Cai, H. Shao, X. Hu, Y. Zhang, Reinforced and ultraviolet resistant silks from silkworms fed with titanium dioxide nanoparticles. ACS Sustain. Chem. Eng. 3, 2551-2557 (2015). https://doi.org/10.1021/acssuschemeng.5b00749

24. Y. Tashiro, T. Morimoto, S. Matsuura, S. Nagata, Studies on the posterior silk gland of the silkworm, Bombyx mori I. Growth of posterior silk gland cells and biosynthesis of fibroin during the fifth larval instar. J. Cell Biol. 38, 574588 (1968). https://doi.org/10.1083/jcb.38.3.574

25. S. Ling, Z. Qi, D.P. Knight, Z. Shao, X. Chen, Synchrotron FTIR microspectroscopy of single natural silk fibers. Biomacromolecules 12, 3344-3349 (2011). https://doi. org/10.1021/bm2006032

26. X. Chen, D.P. Knight, Z. Shao, $\beta$-turn formation during the conformation transition in silk fibroin. Soft Matter 5, 2777-2781 (2009). https://doi.org/10.1039/b900908f

27. H. Pan, Y. Zhang, H. Shao, X. Hu, X. Li, F. Tian, J. Wang, Nanoconfined crystallites toughen artificial silk. J. Mater. Chem. B 2, 1408-1414 (2014). https://doi.org/10.1039/ C3TB21148G

28. T. Asakura, K. Umemura, Y. Nakazawa, H. Hirose, J. Higham, D. Knight, Some observations on the structure and function of the spinning apparatus in the silkworm Bombyx mori. Biomacromolecules 8, 175-181 (2007). https://doi. org/10.1021/bm060874z 
29. L. Cheng, H. Huang, S. Chen, W. Wang, F. Dai, H. Zhao, Characterization of silkworm larvae growth and properties of silk fibres after direct feeding of copper or silver nanoparticles. Mater. Des. 129, 125-134 (2017). https://doi. org/10.1016/j.matdes.2017.04.096

30. D. Nicodemo, J.E. Oliveira, A.A. Sedano, J.M. Marconcini, G.H.D. Tonoli, Impact of different silkworm dietary supplements on its silk performance. J. Mater. Sci. 49, 6302-6310 (2014). https://doi.org/10.1007/s10853-014-8355-4

31. Z. Guo, W. Xie, Q. Gao, D. Wang, F. Gao, S. Li, L. Zhao, In situ biomineralization by silkworm feeding with ion precursors for the improved mechanical properties of silk fiber. Int. J. Biol. Macromol. 109, 21-26 (2018). https://doi. org/10.1016/j.ijbiomac.2017.12.029

32. Z.Z. Shao, F. Vollrath, Surprising strength of silkworm silk. Nature 418, 741 (2002). https://doi.org/10.1038/418741a

33. Q. Wang, C. Wang, M. Zhang, M. Jian, Y. Zhang, Feeding single-walled carbon nanotubes or graphene to silkworms for reinforced silk fibers. Nano Lett. 16, 6695-6700 (2016). https ://doi.org/10.1021/acs.nanolett.6b03597

34. J. Sirichaisit, V.L. Brookes, R.J. Young, F. Vollrath, Analysis of structure/property relationships in silkworm (Bombyx mori) and spider dragline (Nephila edulis) silks using Raman spectroscopy. Biomacromolecules 4, 387-394 (2003). https://doi. org/10.1021/bm0256956

35. S.J. Ling, Q. Wang, D. Zhang, Y.Y. Zhang, X. Mu, D.L. Kaplan, M.J. Buehler, Integration of stiff graphene and tough silk for the design and fabrication of versatile electronic materials. Adv. Funct. Mater. 28, 1705291 (2018). https://doi. org/10.1002/adfm.201705291

36. C. Wang, C. Hsieh, J. Hwang, Flexible organic thin-film transistors with silk fibroin as the gate dielectric. Adv. Mater. 23, 1630-1634 (2011). https://doi.org/10.1002/adma.201004071

37. G. Fang, Z. Zheng, J. Yao, M. Chen, Y. Tang et al., Tough protein-carbon nanotube hybrid fibers comparable to natural spider silks. J. Mater. Chem. B 3, 3940-3947 (2015). https:// doi.org/10.1039/c5tb00448a

38. C. Zhang, Y. Zhang, H. Shao, X. Hu, Hybrid silk fibers dryspun from regenerated silk fibroin/graphene oxide aqueous solutions. ACS Appl. Mater. Interfaces 8, 3349-3358 (2016). https://doi.org/10.1021/acsami.5b11245

39. C. Bao, Y. Guo, L. Song, Y. Hu, Poly(vinyl alcohol) nanocomposites based on graphene and graphite oxide: a comparative investigation of property and mechanism. J. Mater. Chem. 21, 13942-13950 (2011). https://doi.org/10.1039/c1jm11662b

40. J.O. Warwicker, Comparative studies of fibroins II. The crystal structures of various fibroins. J. Mol. Biol. 2, 350-362 (1960). https://doi.org/10.1016/S0022-2836(60)80046-0

41. Y. Liu, L. Yin, H. Zhao, G. Song, F. Tang, L. Wang, H. Shao, Y. Zhang, Insights into process-structure-property relationships of poly(ethylene terephthalate) industrial yarns by synchrotron radiation WAXD and SAXS. J. Appl. Polym. Sci. 132, 42512 (2015). https://doi.org/10.1002/app.42512

42. T. Uemura, S. Kitagawa, Prussian blue nanoparticles protected by poly(vinylpyrrolidone). J. Am. Chem. Soc. 125, 7814-7815 (2003). https://doi.org/10.1021/ja0356582

43. S. Hermes, T. Witte, T. Hikov, D. Zacher, S. Bahnmuller, G. Langstein, K. Huber, R.A. Fischer, Trapping metal-organic framework nanocrystals: an in situ time-resolved light scattering study on the crystal growth of MOF-5 in solution. J. Am. Chem. Soc. 129, 5324-5325 (2007). https://doi.org/10.1021/ ja068835i

44. J. Ren, Y. Liu, D.L. Kaplan, S. Ling, Interplay of structure and mechanics in silk/carbon nanocomposites. MRS Bull. 44, 53-58 (2019). https://doi.org/10.1557/mrs.2018.320

45. S. Gogoi, M. Kumar, B.B. Mandal, N. Karak, High performance luminescent thermosetting waterborne hyperbranched polyurethane/carbon quantum dot nanocomposite with in vitro cytocompatibility. Compos. Sci. Technol. 118, 39-46 (2015). https://doi.org/10.1016/j.compscitech.2015.08.010

46. M. Hu, X. Gu, Y. Hu, T. Wang, J. Huang, C. Wang, Low chemically cross-linked PAM/C-dot hydrogel with robustness and superstretchability in both as-prepared and swelling equilibrium states. Macromolecules 49, 3174-3183 (2016). https ://doi.org/10.1021/acs.macromol.5b02352

47. S. Wu, M. Qiu, Z. Tang, J. Liu, B. Guo, Carbon nanodots as high-functionality cross-linkers for bioinspired engineering of multiple sacrificial units toward strong yet tough elastomers. Macromolecules 50, 3244-3253 (2017). https://doi. org/10.1021/acs.macromol.7b00483

48. N.B. Erdal, M. Hakkarainen, Construction of bioactive and reinforced bioresorbable nanocomposites by reduced nanographene oxide carbon dots. Biomacromolecules 19, 1074 1081 (2018). https://doi.org/10.1021/acs.biomac.8b00207 